\title{
O uso do ácido hialurônico associado ao fechamento de diastema para tratamento de black spaces: estudo de caso clínico
}

\author{
The use of hyaluronic acid associated with diastema closure for the treatment of black \\ spaces: clinical case study \\ El uso de ácido hialurónico asociado al cierre de diastemas para el tratamiento de \\ espacios negros: estudio de caso clínico
}

\begin{abstract}
Samara Kelly da Silva Cavalcante ${ }^{1}$, Tarlóvia Cavalcante Noronha ${ }^{1}$, Álvaro Tavares Lins Roncolato², Karlos Eduardo Rodrigues Lima1, Marcília Ribeiro Paulino², Kéture Kayane Costa Lima1', Talita Arrais Daniel Mendes ${ }^{1,3}$, Sofia Vasconcelos Carneiro ${ }^{1}$, Cosmo Helder Ferreira da Silva ${ }^{1}$ e Natasha Muniz Fontes ${ }^{1 *}$.
\end{abstract}

\section{RESUMO}

Objetivo: Relatar um caso de aplicação clínica do ácido hialurônico associado ao fechamento de diastema com resina composta para o tratamento de black spaces. Detalhamento de caso: Antes do procedimento clínico propriamente dito, verificou-se se o paciente apresentava história médica satisfatória e que não comprometesse a realização do procedimento, após isso, o paciente foi submetido ao clareamento dentário associado, utilizando o gel clareador caseiro à base de peróxido de carbamida a $22 \%$, e o de consultório à base de peróxido de hidrogênio a 35\%. Posteriormente, foi realizado o fechamento de diastema mediano com resina composta seguido de duas aplicações de ácido hialurônico, sendo que as aplicações ocorreram quinzenalmente. Considerações finais: Em suma, neste estudo, o uso de gel de ácido hialurônico injetável associado ao fechamento de diastema com resina composta para tratar black spaces resultou em uma melhora significativa na análise e acompanhamento durante 4 meses, apresentando resultados promissores e de satisfação no paciente.

Palavras-chave: Gengiva, Ácido hialurônico, Diastema.

\begin{abstract}
Objective: To report a case of clinical application of hyaluronic acid associated with diastema closure with composite resin for the treatment of black spaces. Case details: Before the clinical procedure itself, it was verified that the patient had a satisfactory medical history and that it did not compromise the performance of the procedure. After that, the patient was subjected to associated tooth whitening, using the homemade whitening gel based on carbamide peroxide a $22 \%$, and the $35 \%$ hydrogen peroxide-based clinic. Subsequently, the median diastema was closed with composite resin followed by two applications of hyaluronic acid, and the applications occurred every two weeks. Final considerations: In short, in this study, the use of injectable hyaluronic acid gel associated with diastema closure with composite resin to treat black spaces resulted in a significant improvement in the analysis and monitoring for 4 months, presenting promising results and patient satisfaction.
\end{abstract}

Key words: Gingiva, Hyaluronic acid, Diastema.

${ }^{1}$ Centro Universitário Católica de Quixadá (Unicatólica), Quixadá - CE. *E-mail: talita_arrais@hotmail.com

2 Centro Universitário Doutor Leão Sampaio (Unileão), Juazeiro do Norte - CE.

${ }_{3}^{3}$ Programa de Pós-graduação em Odontologia da Universidade Federal do Ceará (UFC), Fortaleza - CE. 


\section{RESUMEN}

Objetivo: Informar un caso de aplicación clínica de ácido hialurónico asociado al cierre de diastemas con resina compuesta para el tratamiento de espacios negros. Detalles del caso: Previo al procedimiento clínico en sí, se comprobó que el paciente tenía un historial médico satisfactorio y que no comprometía la realización del procedimiento. Posteriormente, el paciente fue sometido al blanqueamiento dental asociado, utilizando el gel blanqueador casero a base de peróxido de carbamida un $22 \%$, y la clínica a base de peróxido de hidrógeno al 35\%. Posteriormente, se cerró el diastema medio con resina compuesta seguido de dos aplicaciones de ácido hialurónico, y las aplicaciones se realizaron cada dos semanas. Consideraciones finales: En resumen, en este estudio, el uso de gel de ácido hialurónico inyectable asociado al cierre de diastemas con resina compuesta para el tratamiento de espacios negros resultó en una mejora significativa en el análisis y seguimiento durante 4 meses, presentando resultados prometedores y satisfacción del paciente.

Palabras clave: Encía, Ácido hialurónico, Diastema.

\section{INTRODUÇÃO}

A crescente demanda por reconstruções dentárias esteticamente agradáveis exige que os cirurgiõesdentistas não apenas restaurem a função mastigatória durante o tratamento, mas também criem harmonia visual entre a topografia da gengiva e os contornos gengivais adjacentes. Em particular, a deficiência da papila interdental afeta a estética dos dentes anteriores superiores, bem como a impactação alimentar e a pronúncia das palavras. Além disso, a papila interdental está envolvida em funções fisiológicas complexas como atuar como um escudo biológico para proteger o tecido periodontal. Ademais, uma completa compreensão da interdependência estética dos ossos e tecidos moles, assim como o impacto das aparências relacionadas à papila interdental na estética oral pode facilitar a obtenção de um resultado estético bem-sucedido (ABDELRAOUF AS, et al., 2019).

Os espaços negros, ou black spaces, são definidos como uma perda da papila ao nível cervical em relação ao ponto de contato, causando, portanto, uma imagem inestética, principalmente se ocorrerem ao nível anterior. Sendo de etiologia multifatorial, os black spaces podem ser desencadeados por tratamentos ortodônticos incorretos, angulações incorretas das raízes, coroas dentárias de forma triangular, trauma durante a escovação e principalmente pela periodontite crônica. Além disso, o próprio envelhecimento do paciente tem sido considerado uma causa fisiológica da perda da papila interdental, que ocorre em função do tempo (CHEN M, et al., 2019; ÇANKAYA ZT e TAMAM E, 2020).

Atualmente, várias técnicas, incluindo tratamentos cirúrgicos, ortodônticos, restauradores, protéticos, são usadas para melhorar problemas estéticos relacionados à deficiência da papila interdental. Entre as abordagens invasivas propostas para a reconstrução da papila, a injeção de vários preenchimentos e preparações biológicas foi investigado com ou sem retalho de acesso concomitante. As preparações em gel de ácido hialurônico, muito usadas como preenchimento dérmico, foram recentemente utilizadas para tratar a perda de papila interdental (GULDENER K, et al., 2020).

O Ácido Hialurônico (AH) é um glicosaminoglicano não sulfatado de alto peso molecular, sendo o constituinte mais abundante da matriz extracelular dos tecidos, apresentando papel importante na hidrodinâmica tecidual. Adicionalmente, o AH é um dos principais componentes da matriz do ligamento periodontal, desempenhando funções na adesão, na migração e na proliferação celular. Além disso, possui importantes propriedades anti-inflamatórias, bacteriostáticas e antiedematosas. Quando utilizado na forma injetável, o AH provoca aumento do volume tecidual, contorna e preenche o defeito. Com isso, estudos recentes sugerem a utilização desse material como um possível auxiliar na reconstrução não cirúrgica da papila interdental, por se tratar de um procedimento seguro, minimamente invasivo e bem tolerado pelo paciente (LEE WP, et al., 2016; PILLONI A, et al., 2019).

Contudo, o tratamento de black spaces pode exigir uma abordagem terapêutica restauradora coadjuvante, principalmente em casos de diastemas medianos, que são definidos como um espaçamento da linha média anterior maior que $0,5 \mathrm{~mm}$ entre as superfícies proximais dos incisivos centrais. Resinas compostas diretas 
em casos de fechamento de diastemas permitem aos dentistas o controle completo na formação do sorriso natural. O desenvolvimento de compósitos resinosos com propriedades mecânicas superiores e excelente polibilidade possibilita ao clínico reproduzir a dentição natural e fornecer uma restauração de longa duração ao paciente. Além disso, as resinas compostas permitem um tratamento conservador e, ao mesmo tempo, oferecem resultados mais rápidos (GOYAL A, et al., 2016).

Tendo em vista o que foi exposto, o objetivo deste trabalho foi relatar dois casos de aplicação clínica do $\mathrm{AH}$ associado ao fechamento de diastema com resina composta para o tratamento de black spaces.

\section{DETALHAMENTO DE CASO}

O presente estudo foi autorizado pelo Parecer 4.059.307 do Comitê de Ética do Centro Universitário Católica de Quixadá. Foi incluído paciente maior de 18 anos, que leu e assinou o termo de consentimento livre e esclarecido. Foram excluídos indivíduos com condições médicas que podem afetar a cura ou regeneração periodontal, indivíduos com histórico de reações alérgicas, gestantes ou lactantes, fumantes e alcoólatras. Pacientes com ingestão atual ou prévia de medicamentos que possam predispor ao aumento gengival não foram autorizados a participar. Além disso, não foram selecionados pacientes em tratamento ortodôntico.

O protocolo de tratamento foi dividido em 4 fases: a fase pré-operatória, em que os pacientes foram examinados para elegibilidade, a fase de fechamento de diastema mediano com resina composta, a fase de injeção do AH e a fase de acompanhamento. A terapia periodontal inicial, incluindo raspagem supragengival de boca inteira foi realizada, além da motivação do paciente e educação para instruções de higiene oral adequada foram reafirmadas.

\section{Caso clínico}

Paciente do gênero masculino, 21 anos de idade, normosistêmico, apresenta queixa principal de espaçamento na região dos incisivos centrais superiores, decorrente da deficiência de papila interdental e do ponto de contato dentário está localizado demasiadamente na incisal. Durante o exame clínico intraoral, foi observado deficiência de papila interdental na arcada superior, além da presença de diastema mediano, abrangendo os elementos dentários 11 e 21. Utilizando uma Sonda Milimetrada, verificou-se $5 \mathrm{~mm}$ de black space (Figura 1).

Figura 1 - Aspecto inicial do sorriso do paciente.

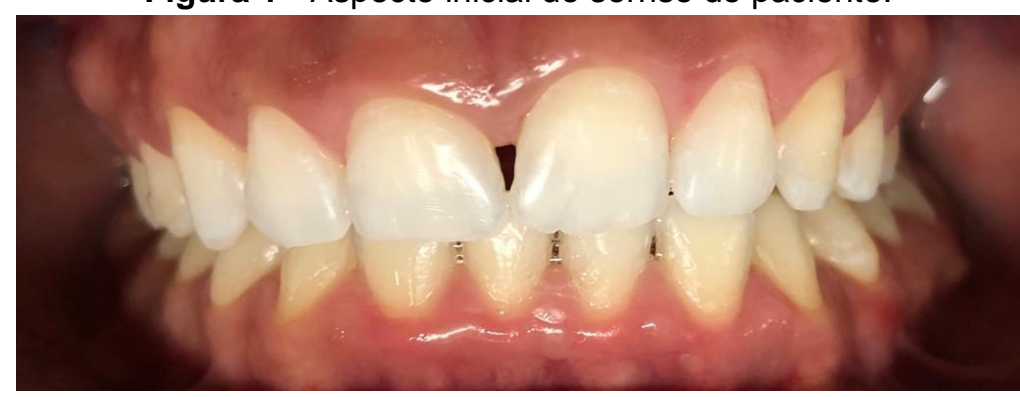

Fonte: Cavalcante SKS, et al., 2021.

Primeiramente, o paciente foi submetido ao clareamento dentário associado, utilizando o gel clareador caseiro à base de peróxido de carbamida a 22\% (Whiteness Perfect, FGM), e o de consultório à base de peróxido de hidrogênio a 35\% (Mix One Supreme, Villevie). Foram realizadas duas sessões do clareamento de consultório e uma sessão de caseiro. Ao final do procedimento, verificou-se uma modificação da cor de A2 para B1, de acordo com a escala Vita Classical. Após o clareamento, aguardou-se um período de aproximadamente duas semanas para dar início ao procedimento restaurador. Para o fechamento de diastema mediano, foi executado tratamento restaurador em sessão única, pela técnica a mão livre (Figura 2). 
Figura 2 - Fechamento de diastema mediano com resina composta.

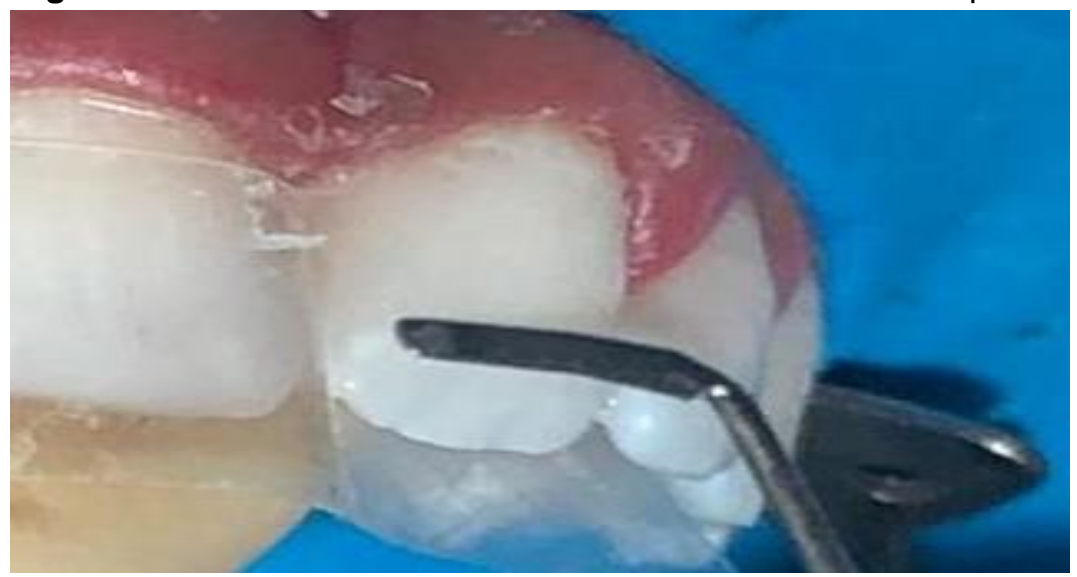

Fonte: Cavalcante SKS, et al., 2021.

Inicialmente, todos os incisivos superiores foram isolados com dique de borracha (Madeitex), posteriormente foi realizada a profilaxia com pedra pomes e água, para melhor seleção de cores da resina composta, sendo selecionadas as cores Translúcidas 20 (IPS EMPRESS DIRECT), A1 corpo (Resina Filtek Z350 XT, 3M ESPE) e B1 esmalte (Resina Filtek Z350 XT, 3M ESPE). Em seguida, o incisivo central adjacente foi coberto com banda de teflon enquanto o outro foi restaurado. O esmalte da superfície mesial foi condicionado com ácido fosfórico a 37\% (AllPrime) por 30 segundos. Removeu-se o ácido com jato de água abundante e foi feita secagem com leves jatos de ar. O sistema adesivo convencional de dois passos (3M ESPE) foi aplicado fazendo esfregaço com o aplicador descartável para evaporação do solvente e polimerizado por 20 segundos.

Uma fina camada de resina composta translúcida foi usada na palatina como esmalte, já como segunda camada foi utilizada a resina opaca. A resina composta de cor A1 foi usada como camada de dentina, e uma camada fina de cor B1 foi usada como a camada superior de esmalte. Cada incremento foi polimerizado com um aparelho a base de LED pelo tempo recomendado pelo fabricante. O mesmo procedimento foi realizado na mesial do incisivo central superior adjacente. Após a remoção do isolamento absoluto, foi realizado acabamento e polimento com tiras de lixa e discos abrasivos de granulação sequencial.

Depois de finalizado o procedimento restaurador, foi realizado duas aplicações de AH (Hialurox Fine 16 $\mathrm{mg} / \mathrm{g}$ ). Previamente, o paciente recebeu anestesia local e anestesias infiltrativas em fundo de sulco anterior da maxila, nas regiões correspondentes aos dentes 11 e 21. Na primeira aplicação, aproximadamente $0,4 \mathrm{ml}$ do gel transparente de AH foi injetado (Figura 3). Após esse tratamento inicial, a injeção de AH foi repetida aos 15 dias, injetando um total de $0,8 \mathrm{ml}$ (Figura 4). No período referente a 3 semanas de acompanhamento, houve percentuais médios de redução de $95 \%$ (Figura 5). Já em uma avaliação de 4 meses de acompanhamento, observou-se um percentual de 100\% de redução (Figura 6).

Figura 3 - Injeção do AH.

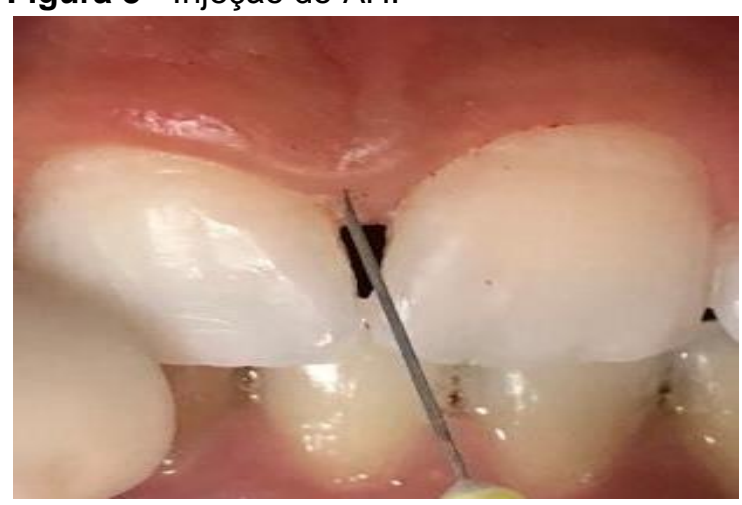

Fonte: Cavalcante SKS, et al., 2021. 
Figura 4 - Aspecto clínico após a aplicação do AH.

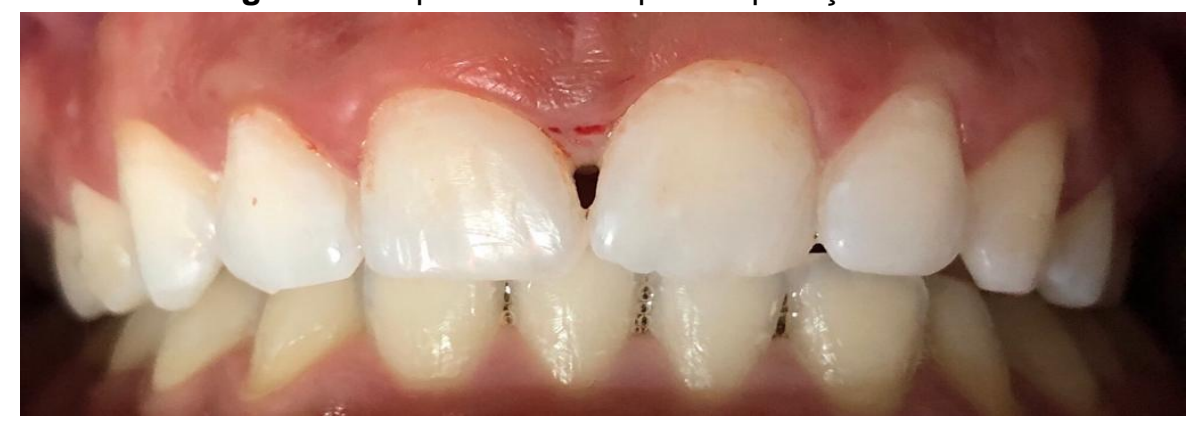

Fonte: Cavalcante SKS, et al., 2021.

Figura 5 - Aspecto final de controle de três semanas da composição do sorriso.

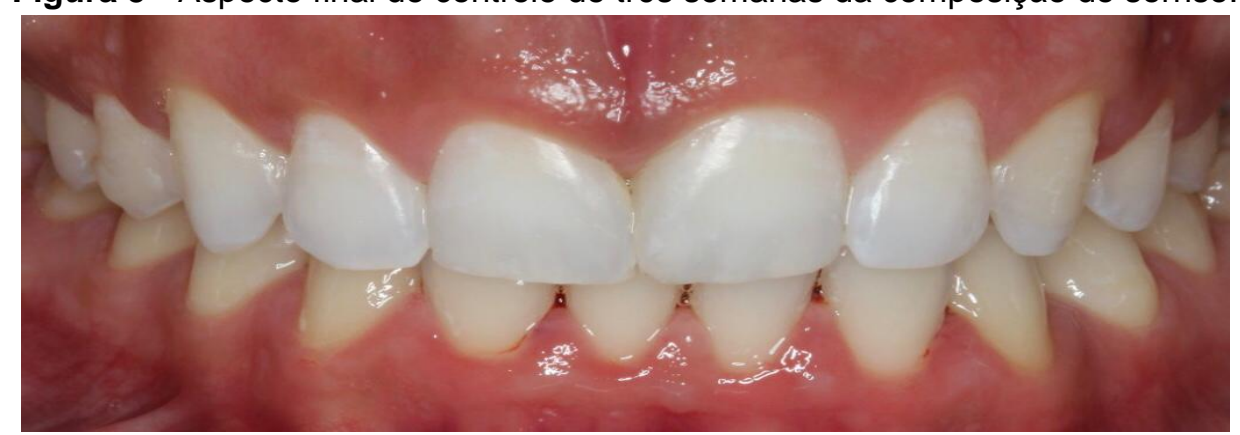

Fonte: Cavalcante SKS, et al., 2021.

Figura 6 - Aspecto final de controle de 4 meses da composição do sorriso.

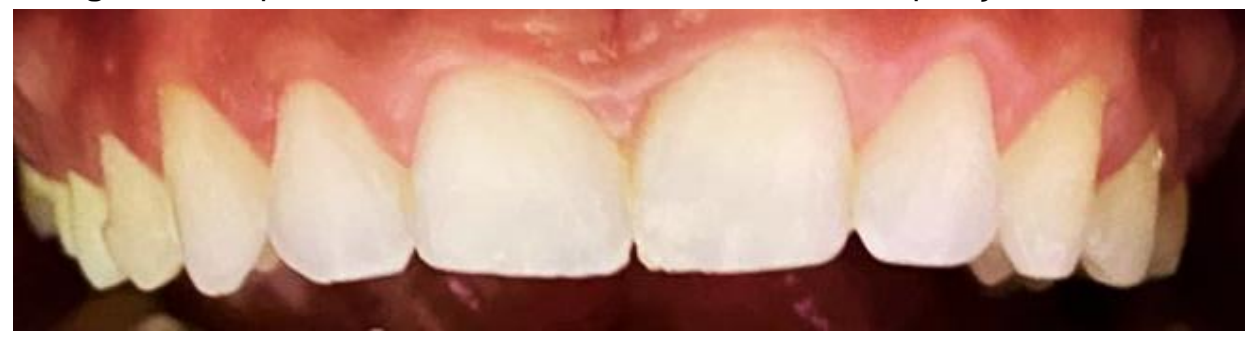

Fonte: Cavalcante SKS, et al., 2021.

\section{DISCUSSÃO}

A porção interdental da gengiva livre, também conhecida como papila interdental, representa uma pequena porcentagem da área de superfície visível dos tecidos duros (dentes) e moles (gengiva, mucosa alveolar), além de apresentar características anatômicas, histológicas e moleculares distintas. Embora aparentemente pequena do ponto de vista anatômico, é grandiosa sob o ponto de vista estético, especialmente na dentição anterior, pois é quase universalmente exibida durante o sorriso. Os black spaces, que se formam com a perda da papila interdental, são classificados como o terceiro problema estético mais desagradável, abaixo da cárie e das margens aparentes da coroa (BERTL K, et al., 2017).

Como a presença de diastema mediano contribui para a ausência de papila interdental, no presente relato de caso, um dos objetivos do tratamento proposto foi a formação de papila com contorno adequado por meio da criação de contatos proximais, além da correção do espaço. Espaços interdentais, ou triângulos pretos, eram evidentes imediatamente após os procedimentos restauradores diretos (SOBRINHO KN, et al., 2016). Conforme recomendado por Kolte AP, et al. (2018), foi realizado um esforço para limitar a distância entre o contato proximal e a crista óssea em $5 \mathrm{~mm}$ ou menos, permitindo que o tecido gengival se reorganizasse nos espaços triangulares. 
No presente caso clínico, a estética foi restabelecida com restaurações diretas de resina composta, nas áreas correspondentes a interproximal dos dentes anteriores superiores. No entanto, Mozayek RS, et al. (2019) relataram que outra opção de tratamento teria sido restaurações cerâmicas indiretas minimamente invasivas. As cerâmicas odontológicas são materiais altamente estéticos e biocompatíveis que oferecem alta dureza e resistência à fratura, bem como excelente resistência ao desgaste. Por outro lado, Ergin $E$, et al. (2018) inferiram que essas restaurações cerâmicas são caras, levando muitos pacientes a recusar esse método de tratamento.

Quando resinas compostas são utilizadas para fechar diastemas, o tratamento é reversível, reparável e modificável, e não há custo biológico, pois não é necessário preparo dentário. Ademais, em comparação com as restaurações indiretas, remodelar os dentes e fechar diastemas com acúmulos de compósitos diretos contemporâneos são vantajosos, especialmente no tratamento de dentes saudáveis em que uma abordagem de economia máxima de tecido é imperativa (KABBACH W, et al., 2018).

Neste relato de caso, ao final do tratamento, as restaurações diretas com resina composta melhoraram significativamente as características anatômicas, ópticas e mecânicas dos elementos dentários tratados, proporcionando um sorriso esteticamente bonito e saudável. Além disso, permitiram que houvesse espaço para a formação da papila interdental na região que abrange os elementos dentários 11 e 21 . Com isso, foi iniciado o tratamento para preenchimento da área de deficiência papilar.

Nesse sentido, Spano SJ, et al. (2020) descreveram que a reconstrução papilar é um dos tratamentos periodontais mais difíceis e desafiadores. Isso ocorre porque a papila interdental é uma área pequena e frágil com pouco suprimento sanguíneo que parece ser o principal fator limitante em todas as técnicas cirúrgicas e de aumento com o objetivo de reconstruir a papila interdental. Em decorrência disso, vários tratamentos têm sido propostos para tratar este estreito espaço e atender às demandas estéticas do paciente, incluindo a injeção de vários preenchimentos (RODRíGUEZ JAM, et al., 2019).

O material usado no presente caso clínico é um gel transparente sem base animal, composto principalmente de $\mathrm{AH}$ de baixo peso molecular, que tem sido usado com sucesso como preenchimento para tratar rugas faciais, plenitude labial insatisfatória e outras consequências do volume inadequado de tecidos moles. O uso de um produto não animal minimiza a possibilidade de reações alérgicas (VANDANA K e SINGH $S, 2019)$. Os black spaces, isto é, a perda das papilas gengivais interdentais, representam uma preocupação estética única originada do volume reduzido de tecido mole intraoral. $\mathrm{O}$ uso de um preenchimento não imunogênico com excelente perfil risco-benefício, como um gel de $\mathrm{AH}$, é uma abordagem não cirúrgica promissora no tratamento da perda de papilas interdentais (NI J, et al., 2019).

Desse modo, a aplicação do AH na área de deficiência papilar, abrangendo os dentes 11 e 21 , foi gradualmente dividida em duas sessões, sendo realizada a primeira aplicação e posterior repetição do procedimento após 15 dias. Foi injetado ao final do tratamento um total de $0,8 \mathrm{ml}$ de AH no caso clinico. Os resultados mostraram que a injeção de gel de AH tem um efeito apreciável no aumento da papila interdental, com regeneração total da área de deficiência papilar.

A porcentagem média de redução na área de superfície do triângulo preto no grupo de $\mathrm{AH}$ no presente estudo foi de $100 \%$ da linha de base a 4 meses de acompanhamento. Esses resultados são consistentes com um estudo conduzido anteriormente por Mansouri SS, et al. (2013) que relatou percentuais médios de redução de $29,52 \pm 18,72 \%$ no período de referência a 3 meses e $47,33 \pm 20,20 \%$ no período de referência a 6 meses. Em concordância com o presente estudo, a porcentagem média de redução em outro estudo realizado por Awartani FA e Tatakis DN (2015) foi de $41 \pm 37 \%$ após 6 meses. Aliás, em uma série de casos desenvolvida por Becker W, et al. (2010) também foi relatada uma porcentagem média de redução de $91,1 \pm$ 11,99\% após 25 meses de acompanhamento da aplicação do gel de $\mathrm{AH}$. Eles não forneceram quaisquer dados sobre área de superfície do triângulo preto no início do estudo, mas apenas recomendaram a injeção de gel de $\mathrm{AH}$ em pequenos defeitos papilares. A maior porcentagem de redução em seu estudo é provavelmente atribuída ao tamanho menor dos black spaces na linha de base e ao período de acompanhamento mais longo. 
Além disso, Lee WP, et al. (2016), através de outra série de casos, registraram um percentual médio de redução de $92,55 \% \pm 13,46 \%$ após o acompanhamento de 6 meses de tratamento. Ao contrário dos estudos acima, Bertl K, et al. (2017) não encontraram diferenças significativas nos grupos de injeção de teste (injeção de $\mathrm{AH}$ ) e de controle (injeção de solução salina), nem no início do estudo, nem em 3 e 6 meses após o tratamento. A inclusão do grupo de controle, por meio da injeção de solução salina, é altamente questionável. Nenhum dos estudos acima observou deficiência de papila interdental por meio de medição clínica.

Considerando as limitações do estudo, concluiu-se que o uso do gel de $\mathrm{AH}$ injetável associado ao fechamento de diastema com resina composta permitiu uma reconstrução bem-sucedida, com níveis promissores de satisfação do paciente. Essa abordagem terapêutica dispensa o uso de procedimentos cirúrgicos para regenerar a papila perdida, portanto, é um método não invasivo, reduzindo o desconforto do paciente. Para superar as limitações do uso do $\mathrm{AH}$ associado ao fechamento de diastema como tratamento de black spaces, este relato de caso precisa ser elaborado com maior número de pacientes e dependendo do tamanho do black space.

\section{REFERÊNCIAS}

1. ABDELRAOUF SA, et al. Assessment of Hyaluronic Acid Gel Injection in the Reconstruction of Interdental Papilla: a randomized clinical trial. Open Access Macedonian Journal of Medical Sciences, 2019; 7(11): 1834-1840.

2. AWARTANI FA, TATAKIS DN. Interdental papilla loss: treatment by hyaluronic acid gel injection: a case series. Clin Oral Investig, 2015; 7: 1775-1780.

3. BECKER W, et al. Minimally invasive treatment for papillae deficiencies in the esthetic zone: a pilot study. Clin Implant Dent Relat Res, 2010; 12(1): 1-8.

4. BERTL K, et al. Can hyaluronan injections augment deficient papillae at implant-supported crowns in the anterior maxilla? A randomized controlled clinical trial with 6 months' follow-up. Clinical Oral Implants Research, 2017; 28(9): 1054-1061.

5. ÇANKAYA ZT, TAMAM E. An examination of the 2-year results obtained from hyaluronic acid filler injection for interdental papilla losses. Quintessence International, 2020; 51(4): 274-284.

6. CHEN M, et al. High molecular weight hyaluronic acid regulates $\mathrm{P}$. gingivalis-induced inflammation and migration in human gingival fibroblasts via MAPK and NF-KB signaling pathway. Archives of Oral Biology, 2019; 98: 75-80.

7. ERGIN E, et al. Comparison of two different composite resins used for tooth reshaping and diastema closure in a 4year follow-up. Niger J Clin Pract, 2018; 21(9): 1098-1106.

8. GOYAL A, et al. Diastema Closure in Anterior Teeth Using a Posterior Matrix. Case Reports In Dentistry, 2016; 2016: 1-6.

9. GULDENER K, et al. Treatment of single mandibular recessions with the modified coronally advanced tunnel or laterally closed tunnel, hyaluronic acid, and subepithelial connective tissue graft: a report of 12 cases. Quintessence Int, 2020; 51(5): 456-463.

10. KABBACH W, et al. Diastema closures: a novel technique to ensure dental proportion. Journal Of Esthetic And Restorative Dentistry, 2018; 30(4): 275-280.

11. KOLTE AP, et al. Proximal contact areas of maxillary anterior teeth and their influence on interdental papilla. The Saudi Dental Journal, 2018; 30(4): 324-329.

12. LEE WP, et al. Six Month Clinical Evaluation of Interdental Papilla Reconstruction with Injectable Hyaluronic Acid Gel Using an Image Analysis System. Journal of Esthetic and Restorative Dentistry, 2016; 28(4): 221-230.

13. MANSOURI SS, et al. Clinical Application of Hyaluronic Acid for Reconstruction of Interdental Papilla at the Esthetic zone. The Journal of Islamic Dental Association of IRAN (JIDA), 2013; 25(2): 152-157.

14. MOZAYEK RS, et al. Porcelain sectional veneers, an ultra-conservative technique for diastema closure (threedimensional finite element stress analysis). Dental and Medical Problems, 2019; 56(2): 179-183.

15. NI J, et al. Efficacy Evaluation of Hyaluronic Acid Gel for the Restoration of Gingival Interdental Papilla Defects. Journal of Oral and Maxillofacial Surgery, 2019; 77(12): 2467-2474.

16. PILLONI A, et al. Effectiveness of adjunctive hyaluronic acid application in coronally advanced flap in Miller class I single gingival recession sites: a randomized controlled clinical trial. Clinical Oral Investigations, 2019; 23(3): 11331141.

17. RODRÍGUEZ JAM, et al. Periodontal reconstructive surgery of deep intraosseous defects using an apical approach. Non-incised papillae surgical approach (NIPSA): a retrospective cohort study. Journal of Periodontology, 2018; 90(5): 454-464.

18. SOBRINHO KN, et al. The role of emergence profile in papilla maintenance after diastema closure with direct composite resin restorations. Gen Dent, 2016; 64(3): 1-4.

19. SPANO SJ, et al. Subperiosteal Papilla Augmentation with a Non-Animal-Derived Hyaluronic Acid Overlay Technique. Clinical Advances in Periodontics, 2020; 10(1): 4-9.

20. VANDANA K, SINGH S. Use of different concentrations of hyaluronic acid in interdental papillary deficiency treatment: a clinical study. Journal of Indian Society of Periodontology, 2019; 23(1): 35-41. 